Review Article

\title{
Targeting the Glutamatergic System to Treat Pathological Gambling: Current Evidence and Future Perspectives
}

\author{
Mauro Pettorruso, ${ }^{1}$ Luisa De Risio, ${ }^{1}$ Giovanni Martinotti, ${ }^{2}$ Marco Di Nicola, \\ Filippo Ruggeri, ${ }^{1}$ Gianluigi Conte, ${ }^{1}$ Massimo Di Giannantonio, ${ }^{2}$ and Luigi Janiri ${ }^{1}$ \\ ${ }^{1}$ Institute of Psychiatry and Psychology, Catholic University of the Sacred Heart, Largo Agostino Gemelli 8, 00168 Rome, Italy \\ ${ }^{2}$ Department of Neuroscience and Imaging, "G. d'Annunzio" University, Chieti, Italy
}

Correspondence should be addressed to Mauro Pettorruso; mauro.pettorruso@hotmail.it

Received 28 February 2014; Accepted 22 May 2014; Published 12 June 2014

Academic Editor: Sophia Achab

Copyright (C) 2014 Mauro Pettorruso et al. This is an open access article distributed under the Creative Commons Attribution License, which permits unrestricted use, distribution, and reproduction in any medium, provided the original work is properly cited.

\begin{abstract}
Pathological gambling or gambling disorder has been defined by the DSM-5 as a behavioral addiction. To date, its pathophysiology is not completely understood and there is no FDA-approved treatment for gambling disorders. Glutamate is the principal excitatory neurotransmitter in the nervous system and it has been recently involved in the pathophysiology of addictive behaviors. In this paper, we review the current literature on a class of drugs that act as modulating glutamate system in PG. A total of 19 studies have been included, according to inclusion and exclusion criteria. Clinical trial and case series using glutamatergic drugs $(\mathrm{N}$-acetylcysteine, memantine, amantadine, topiramate, acamprosate, baclofen, gabapentin, pregabalin, and modafinil) will be presented to elucidate the effectiveness on gambling behaviors and on the related clinical dimensions (craving, withdrawal, and cognitive symptoms) in PG patients. The results have been discussed to gain more insight in the pathophysiology and treatment of PG. In conclusion, manipulation of glutamatergic neurotransmission appears to be promising in developing improved therapeutic agents for the treatment of gambling disorders. Further studies are required. Finally, we propose future directions and challenges in this research area.
\end{abstract}

\section{Background}

Pathological gambling (PG) is characterized by persistent and maladaptive gambling behavior, whereby individuals engage in frequent and repeated episodes of gambling despite serious adverse consequences [1]. Gambling disorder affects $0.2-$ $5.3 \%$ of adults worldwide; the devastating consequences of this behavioral disturbance often entail severe damage to the lives of patients and their families. To date, there is no FDA-approved treatment for PG, despite almost a decade of intense research, and effective treatment strategies remain very challenging. Recently, PG has been included in the diagnostic category of substance use and addictive disorders in the 5th edition of the Diagnostic and Statistical Manual of Mental Disorders (DSM-V).

Glutamate (Glu) is the principal excitatory neurotransmitter in the nervous system. It has been recently proposed that addiction can be viewed as the result of an impaired ability to inhibit drug seeking in response to environmental contingencies, due to alterations in Glu homeostasis, with combined activation of sensitized dopamine (DA) and Nmethyl-d-aspartate (NMDA) glutamatergic receptors [2]. Blocking the release of Glu has prevented drug seeking behaviors in animals as well as patients with substance use disorders $[3,4]$. The clinical and biological similarities between PG and drug addiction [5] suggest that PG patients may benefit from medication used to treat drug addiction and that pathophysiological models for drug addiction may be relevant to PG as well.

In this paper, we review the current literature on drugs that modulate glutamatergic neurotransmission in PG. We also elucidate current hypotheses on the neurobiology of PG, focusing on glutamatergic neurotransmission and its interactions with other neurotransmitters. Clinical trials and 
case series using glutamatergic drugs will be presented to elucidate the effectiveness on gambling behaviors and on the related clinical dimensions (craving, withdrawal, and cognitive symptoms) in PG patients. The results will be discussed to gain more insight into the pathophysiology and treatment of PG. Finally, we propose future directions and challenges in this research area.

\section{Methods}

Two reviewers were separately engaged in this review, following the same bibliographic search and data extraction protocol. Bibliographic search consisted of a computerized screening of Medline, Scopus, and Google Scholar database in January 2014. Only English language studies published in the last ten years were reviewed. We used the following queries: "gambl*" combined with "glutamate" and with a list of glutamatergic neurotransmission-modulating agents including $\mathrm{N}$-acetylcysteine, memantine, amantadine, acamprosate, topiramate, lamotrigine, baclofen, gabapentin, pregabalin, modafinil, riluzole, dizocilpine, LY354740, D-cycloserine, methadone, and dextromethorphan. The search initially yielded 99 results. We then hand-searched relevant references of each article, including earlier studies on the topic.

Of the 99 potential articles, 19 were included (Figure 1) according to the following criteria: (a) the target problem is PG; (b) the abstract is available; (c) the publication is an original paper, excluding reviews; (d) the study is a neurobiological or a clinical research on PG subjects.

Table 1 shows relevant data from the articles included in the study: drug used, dosage, study design, sample size and targeted population, methods, cognitive outcome, and main finding on gambling outcome.

\section{Glutamatergic Transmission in Addictive Behaviors: Relevance for Pathological Gambling}

Glu is the most prevalent excitatory neurotransmitter in the CNS and its action is regulated by two types of receptors: the ionotropic (iGlu) and metabotropic (mGlu) receptors. The ionotropic receptors are ion channels that, upon Glu binding, increase the influx of sodium and potassium cations causing depolarization of the membrane [19]. They are divided into three subtypes: N-methyl-D-aspartate (NMDA), $\alpha$-amino3-hydroxy-5-methyl-4-isoazole-propionic acid (AMPA), and kainate. The metabotropic receptors are $G$ protein-coupled receptors and are divided into three groups (I, II, and III) based on the homology of the sequences, the mechanism of signal transduction, and their pharmacological selectivity [20]. The metabotropic receptors are located primarily in the limbic and frontal areas, which are specifically involved in the mechanisms of addiction. In particular, receptors of group I seem to have an important role in the regulation of the reinforcing effects of drugs, while type II receptors are implicated in synaptic changes that occur as a result of prolonged exposure to the drug and in withdrawal syndromes [21]. Following abuse of any substance, increased glutamatergic transmission occurs in the limbic system and the prefrontal cortex which seems to be responsible, first and foremost, a greater release of DA, and also DA-dependent effects. In particular, while phenomena such as sensitization, craving, relapse, and reinforcement are linked to changes in both dopaminergic and glutamatergic systems, specific context and conditioned behaviors related to substance use primarily depend on glutamatergic mechanisms [22]. Summarily, the glutamatergic-dopaminergic system (in the nucleus accumbens) is responsible for the onset of "drug seeking," while relapse only involves the glutamatergic system [23]. Reduction of extracellular glutamate levels in the limbic areas seems to be closely related to the withdrawal syndrome from psychostimulants; metabotropic glutamate receptor agonists seem to be able to reduce craving and prevent relapse via a compensation mechanism. Also, antagonists of metabotropic receptors hinder the behavioral effects of cocaine, nicotine, and alcohol, and NMDA antagonists are potential candidates for the treatment of opiate, alcohol, and sedative withdrawal syndromes [24].

PG has been presumed to be modulated mainly by brain DA and Glu, though findings are contrasting. DA is implicated in rewarding, reinforcing, and addictive behaviors. In drug addiction, data support the existence of a hypodopaminergic state at both the presynaptic and postsynaptic levels [25]. While DA release may reinforce learning [26, 27], Glu may be implicated in long-lasting neuroadaptations in the corticostriatal circuitry that represents the putative neural substrate of enduring vulnerability to relapse [2]. Glu is involved in learning and memory and may activate different types of Glu receptors, including NMDA receptors expressed in brain regions comprising reward circuitry [2]. Levels of Glu within the nucleus accumbens mediate rewardseeking behavior [2]. Pathological gamblers report euphoric feelings during gambling episodes, comparable to the "high" in substance use, thus making them more prone to continued gambling. In addition, preliminary reports showed a reduction of hedonic capacity in response to stimuli usually perceived as rewarding [28]. By continued gambling, the salience attribution to the behavior is strengthened and induces cue reactivity which can result in craving phenomena and potentially further enhancement of DA neurotransmission. Finally, continued gambling and subsequent altered DA neurotransmission could lead to neuroadaptation in mesolimbicprefrontal glutamatergic pathways [29]. Chronic drug intake is associated with neuroadaptation of glutamatergic neurotransmission in the ventral striatum and limbic cortex [30]. In addition, cue exposure has been found to depend on strong projections of glutamatergic neurons from the prefrontal cortex to the nucleus accumbens [31]. Repetitive behaviors closely followed by rewards increase extracellular Glu levels [32]. In one study, cerebrospinal fluid (CSF) levels of glutamic and aspartic acid, both of which bind to NMDA receptors, were elevated among PG patients as compared to control subjects [33]. The imbalance in Glu homeostasis engenders changes in neuroplasticity which impair communication between the prefrontal cortex and the nucleus accumbens, thus favoring engagement in reward-seeking behaviors, such as PG [34]. 


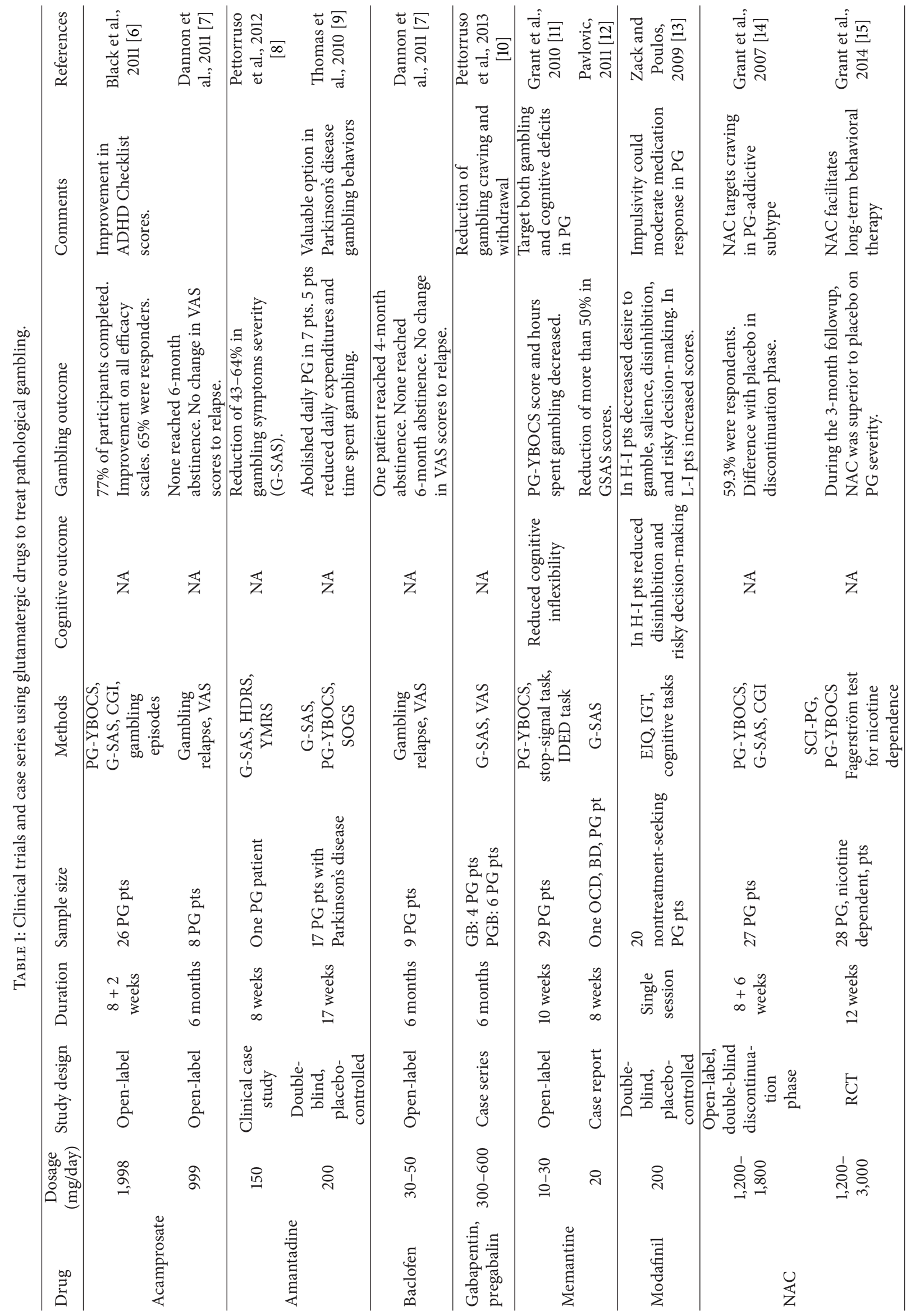




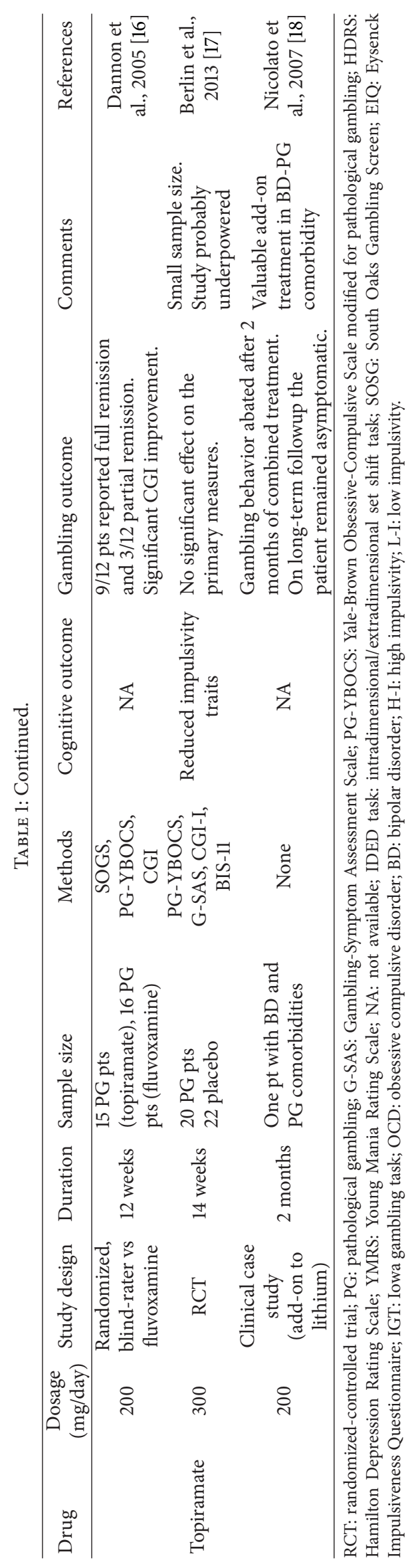




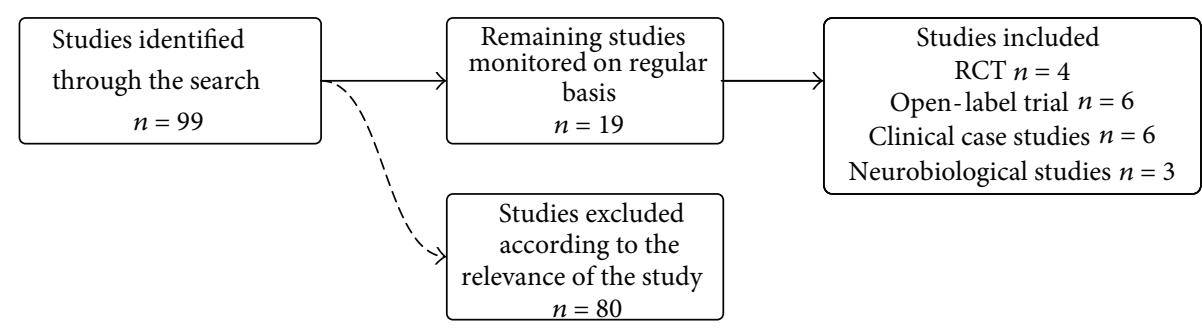

FIGURE 1: Bibliographic process.

\section{Glutamatergic Treatment Strategies in Pathological Gambling}

Manipulation of glutamatergic neurotransmission is a relatively young but promising avenue for the development of improved therapeutic agents for the treatment of drug and behavioral addictions $[10,35]$. Substantial evidence has accumulated indicating that ligands acting on glutamatergic transmission are also of potential utility in the treatment of drug addiction, as well as various behavioral addictions such as pathological gambling. Growing evidence suggests that the glutamatergic system is central to the neurobiology and treatment of mood disorders [36] and that it could represent a valuable target in PG with comorbid conditions [37].

4.1. N-Acetylcysteine. N-Acetylcysteine (NAC), a cysteine prodrug and amino acid, can increase extracellular levels of Glu concentration in the nucleus accumbens and has shown preliminary efficacy in treating substance addictions [38, 39]. NAC may stimulate inhibitory metabotropic Glu receptors, possibly causing a reduction in synaptic release of glutamate. Studies in rat populations show that NAC is effective in reducing reward-seeking behavior [40] and preliminary data in $\mathrm{PG}$ are encouraging.

NAC was found to be effective in reducing gambling urges and behavior (lower scores on the Yale-Brown Obsessive Compulsive Scale modified for PG (PG-YBOCS)) in a small clinical trial [14]. Twenty-seven PG subjects (12 women) were treated for 8 weeks with NAC (mean dose was 1476.9 $\pm 311.3 \mathrm{mg} /$ day). Responders were randomized in a 6-week double-blind discontinuation trial (NAC vs placebo). A significantly higher percentage of subjects treated with NAC still meet responder criteria at the end of the study $(83.3 \%$ in NAC versus $28.6 \%$ in placebo group). In addition, a recent RCT confirmed the efficacy of NAC augmentation of behavioral therapy in the treatment of PG [15]. The study was conducted on 28 subjects with cooccurring nicotine dependence and PG. They received behavioral therapy and were randomized to augmentation with NAC (up to 3,000 mg/day) or placebo in a double-blind trial. During the final 3-month followup, there was a significant additional benefit for NAC versus placebo on gambling severity measures (PG-YBOCS).

Several matters remain unresolved. The optimal dose of NAC for PG is still unknown. The dose used in the augmentation-RCT was notably higher than that used in the previous study. According to preclinical data in rats, lower concentrations of NAC inhibit Glu transmission in the nucleus accumbens core while higher concentrations countermand this effect [41]. Given NAC glutamatergic properties and glutamate's role in learning and memory in addictive processes [42], its use has been proposed for patients who report craving to gamble and for those who are also undergoing an exposure-based psychosocial intervention.

4.2. Memantine. Memantine, a noncompetitive antagonist at the NMDA receptor with neuroprotective properties, is approved for Alzheimer's disease and is increasingly being studied in a variety of psychiatric disorders [43]. In PG patients memantine decreased PG-YBOCS scores and time spent gambling, also improving neurocognitive function related to cognitive flexibility [11]. Twenty-nine subjects were enrolled in a 10-week open-label trial. After memantine treatment (10-30 mg/day), PG-YBOCS scores and hours spent gambling decreased significantly. In addition, subjects underwent pre- and posttreatment cognitive assessment using the stop-signal task and the intradimensional/extradimensional (IDED) set shift task to assess impulsivity and cognitive flexibility, respectively. At study endpoint, a significant improvement in IDED performance was found, probably due to memantine modulation of glutamatergic transmission in PFC [44]. Nonetheless, the extent to which memantine exerts its influences on gambling behaviors through effects on impulsivity or compulsivity is still unclear [45].

A clinical case study reports effectiveness of memantine in the treatment of a 23-year-old patient with obsessivecompulsive disorder, body dysmorphic disorder, and severe PG [12]. A clinical response was observed after 8 weeks of memantine treatment, with more control over gambling and less anticipatory tension and excitation.

Memantine seems to reduce Glu excitability and improve impulsive decision-making. In addition, it shows promise in the treatment of cognitive and compulsive symptoms in PG patients $[11,45]$.

4.3. Amantadine. Amantadine, an antiglutamatergic drug with additional actions on dopaminergic neurotransmission, has been evaluated in treating PG and other compulsive behaviors in individuals with Parkinson's disease [9, 46]. Conflicting data have been reported regarding use of amantadine among Parkinson's disease patients [47]. It was found to be safe and effective in 17 patients with PG, reducing or stopping gambling urges and behaviors [9]. In a cross-sectional 
study amantadine was associated with PG and other impulse control disorders [48].

In addition, a case study suggested the possible utility in the treatment of PG patients [8]. A significant improvement on gambling symptoms suggests that simultaneous pharmacological modulation of the glutamatergic and dopaminergic systems may reduce gambling in PG, possibly reversing neuroplasticity-based pathological changes determined by addictive behaviors [2].

4.4. Topiramate. Topiramate is a glutamatergic antagonist and pro-GABAergic drug that significantly reduces impulsive behavior and compulsiveness. It has been tested and found to be effective versus placebo in disorders in which impulsivity and craving represent core features, such as alcohol dependence, cocaine dependence, bulimia nervosa, and binge eating disorder. In addition, it has recently been proposed that topiramate is also an antagonist of AMPA receptors, a Glu receptor subtype that mediates relapse-like behaviors and is implicated in the neuroadaptive changes produced by drugs of abuse as well [49].

A 14-week, randomized, double-blind, placebo-controlled trial investigated topiramate in PG [17]. Though no significant differences between the placebo group and the topiramate-treated group were observed with respect to primary outcome measures (change in the obsessions subscale of the PG-YBOCS), topiramate reduced impulsivity (particularly, motor and nonplanning impulsivity), as measured with the Barratt Impulsiveness Scale (BIS). The authors suggest that topiramate could be useful in PG subgroups characterized by high levels of impulsivity. Dannon et al. [16] compared the effectiveness of topiramate versus fluvoxamine in the treatment of PG in a 12-week, blind-rater comparison trial. Though the authors conclude that both topiramate and fluvoxamine monotherapies may be effective in the treatment of PG, improvement on the PG-CGI for fluvoxamine did not quite reach statistical significance. Also, a smaller number of dropouts were reported in the topiramate group.

In addition, in a patient with bipolar disorder and comorbid PG, Nicolato et al. [18] reported full remission of gambling craving and behavior after topiramate was added to standard lithium treatment.

4.5. Acamprosate. Acamprosate (calcium acetylhomotaurinate) is a taurine derivative and a nonspecific GABA agonist that promotes a balance between excitatory and inhibitory neurotransmitters (Glu and GABA). It binds specifically to GABAB receptors and appears to block Glu receptors and inhibit hyperactive glutamatergic signaling [50]. Although there is accumulated evidence suggesting that acamprosate interferes with the Glu system by antagonizing NMDA receptor activity [51], its mechanism of action still remains unclear. Recent findings suggest the involvement of calciummediated pathways [52]. These inconsistencies are perhaps related to factors such as brain region examined, NMDA receptor subunit composition, state of neuronal excitation, and the presence of various endogenous NMDA receptor neuromodulators such as polyamines $[50,53]$. Acamprosate has been approved by the FDA for alcohol dependence.
Restoring the imbalance between excitatory and inhibitory neurotransmissions caused by chronic alcohol exposure [53], it has been found to raise the continuous alcohol abstinence rate and double the days of cumulative abstinence from alcohol [54].

Contrasting results have been reported on its use in PG treatment [55]. In an 8-week, open-label trial following a 2-week observation, acamprosate significantly improved PG-YBOCS and Gambling Severity Assessment Scale (GSAS) scores, both CGI scales, and number of gambling episodes [6]. Twenty-six patients received the medication $(1,998 \mathrm{mg} /$ day $)$. The primary efficacy measure was the PGYBOCS. Secondary efficacy measures included the G-SAS, the Clinical Global Impression (CGI) Improvement and Severity scales, a patient self-rated global rating, the Hamilton Depression Rating Scale (HDRS), the Sheehan Disability Scale (SDS), and the timeline follow back (TLFB).

In contrast, a parallel study failed to confirm its effectiveness on gambling behavior [7]. In this open-label study, 8 pathological gamblers treated with acamprosate $999 \mathrm{mg} /$ day were evaluated monthly for 6 months to assess relapse. None of the patients attained 6 months of abstinence, defined as the absence of any gambling behavior during the month preceding the follow-up visit. VAS scores at baseline, after 1 month, and at relapse showed no statistically significant differences. No validated scales were employed to determine the effectiveness of acamprosate on gambling urges and craving.

4.6. Baclofen. Baclofen (beta-(4-chlorophenyl)-GABA) is a GABAB receptor agonist that has been found to suppress both acquisition of alcohol drinking behaviors in rats and daily alcohol intake in alcohol experienced rats. By inhibiting multivesicular release from the presynaptic terminal, it decreases synaptic Glu signaling [56] and inhibits Ca2+ permeability of NMDA receptors. In rats, it also suppresses alcohol-stimulated dopamine release in the shell of the nucleus accumbens [57].

In an open-label trial [7], 9 patients receiving baclofen were evaluated on a monthly basis in order to assess measures of sustained improvement (i.e., abstinence) and relapse. None of the patients attained 6 months of abstinence, defined as the absence of any gambling behavior during the month preceding the follow-up visit; only one patient receiving baclofen attained 4 months of abstinence. VAS scores at baseline, after 1 month, and at relapse showed no statistically significant differences.

4.7. Gabapentin and Pregabalin. Anticonvulsants, like gabapentin and pregabalin, have multiple mechanisms of action, including inhibition of presynaptic voltage-gated $\mathrm{Na}+$ and $\mathrm{Ca} 2+$ channels, thereby inhibiting the relapse of neurotransmitters including glutamate. Gabapentin modulates both GABAergic and glutamatergic neurotransmissions. Several authors have explored the use of gabapentin in substance use disorders. Gabapentin reverses GABA deficits and Glu excess thought to underlie alcohol withdrawal and early abstinence. It reduces alcohol consumption and craving, thereby facilitating abstinence [58]. Pregabalin is a structural analog of GABA, similar to gabapentin. It also reduces excitatory 
neurotransmitter release and postsynaptic excitability. The FDA has approved pregabalin for partial epilepsy, neuropathic pain, and generalized anxiety disorders. In addition, pregabalin has been extensively studied in alcohol and benzodiazepine dependence [59]. A 6-month pilot trial preliminarily investigated the potential utility of their use in PG patients (6 patients received pregabalin; 4 patients received gabapentin), with a reduction of gambling craving as measured by G-SAS [10]. Also, pregabalin has been used to treat a case of citalopram-associated gambling onset [60]. Future studies should investigate the use of gabapentin and pregabalin in the treatment of PG, given that this drug seems to specifically target the central features of impulsivity, anxiety, and craving.

4.8. Modafinil. Modafinil is an atypical stimulant, originally designed to enhance wakefulness and vigilance in the treatment of narcolepsy and sometimes prescribed as an off-label treatment for attention-deficit/hyperactivity disorder (ADHD). Although its mechanisms of action are not completely understood, modafinil does not appear to act as a monoamine releaser as is the case for amphetaminelike stimulants. Rather, modafinil may act by stimulating $\alpha$ adrenoceptors, suppressing GABA release, weakly inhibiting the dopamine transporter, or stimulating hypothalamic orexin-containing neurons $[61,62]$. While most studies suggest a dopaminergic basis for its stimulant effects [63], modafinil has been shown to elevate extracellular levels of glutamate in numerous brain regions including the dorsal striatum, hippocampus, and diencephalon without affecting glutamate synthesis $[35,64]$. Numerous clinical reports have shown that modafinil demonstrates potential efficacy in the treatment of cocaine addiction [62].

Zack and Poulos [13], in a placebo-controlled doubleblind trial, tried to determine if modafinil (mean dose $200 \mathrm{mg} /$ day) reduces the reinforcing effects of slot machine gambling in PG subjects and if this effect is stronger in high versus low impulsivity subjects $(N=20)$. Bet size declined uniformly in both high and low impulsivity participants taking modafinil. In high impulsivity participants, modafinil decreased desire to gamble, salience of gambling words, disinhibition, and risky decision-making. In low impulsivity participants, modafinil increased scores on these indices. The results showed that modafinil had bidirectional effects in the two groups. The same sample of patients was reevaluated in a prospective study, with clinical results highlighting that modafinil may discourage pathological gamblers from chasing losses but also encourage them to continue betting, rather than quitting while they are ahead [65]. Also, it has been reported a case of clear-cut temporal relationship between modafinil treatment and pathological gambling in a 39year-old patient with a history of narcolepsy and associated cataplexy [66].

\section{Discussion}

There is substantial evidence indicating that pharmacological treatments targeting glutamatergic transmission are of potential utility in the treatment of drug addiction. Given that neurobiological findings indicate that PG and drug addiction share common etiopathological pathways $[5,45]$, drugs targeting glutamatergic transmission could be of use for the treatment of behavioral addictions (i.e., PG) as well.

The data seem to confirm the utility of targeting the glutamatergic system for the treatment of PG, in particular by acting on craving and increasing treatment retention $[10,15]$. Glutamatergic medications may, in fact, offer some advantages in preventing relapse [4]. It has been recently proposed that addiction can be viewed as the result of an impaired ability to inhibit drug seeking in response to environmental contingencies, due to alterations in Glu homeostasis, with combined activation of sensitized DA and NMDA glutamatergic receptors [2]. Glutamatergic drugs may regulate the complex interactions between the glutamatergic and dopaminergic systems, acting simultaneously on both systems, in ways that need to be better explored.

Studies discussed are not homogeneous with respect to the criteria used to evaluate the effectiveness of pharmacological treatments for PG. In fact, some studies consider the absence of gambling behavior as the primary outcome while overlooking important clinical dimensions including craving and withdrawal symptoms. Interestingly, research on glutamatergic drugs highlights the importance of pointing clinical attention to the detection and treatment of cognitive symptoms [29]. Pathological gamblers exhibit a pattern of decision-making that repeatedly ignores long-term negative consequences in order to obtain immediate gratification or relief from uncomfortable states associated with their addiction. A variety of cognitive and emotional processes influence decision-making [11]. These alterations (i.e., cognitive inflexibility) may contribute to deviant choice in PG patients and to the maintenance of the disorder, as indirectly confirmed by the potential efficacy of cognitive therapy focused on altering irrational gambling cognition [67]. Targeting this clinical dimension, throughout the pharmacological modulation of the glutamatergic system, could be a useful treatment perspective and needs further study.

Drugs that enhance decision-making and executive function abilities are less well known because of the complexity of these functions which comprise different subprocesses (i.e., reward, punishment sensitivity, and impulsivity). However, it can be argued that agents targeting these subprocesses may improve decision-making as well. In addition, cognitive enhancers such as modafinil might also have beneficial effects, particularly in high impulsivity subjects [13].

\section{Future Perspectives}

The data seem to confirm the utility of targeting the glutamatergic system for the treatment of PG, in particular by acting on craving and cognitive domains (impulsivity and cognitive inflexibility). While empirically validated treatments for PG have varying degrees of support, little is known about their mechanisms of action or how specific therapies might work better for specific individuals. Several studies have been conducted to test the efficacy of opioid antagonists in the treatment of the disorder, and a genetic predisposition or a family history of alcoholism has been hypothesized 


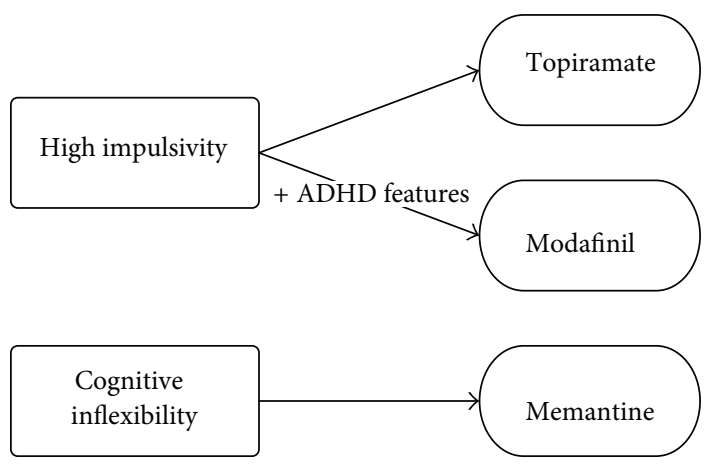

(a)

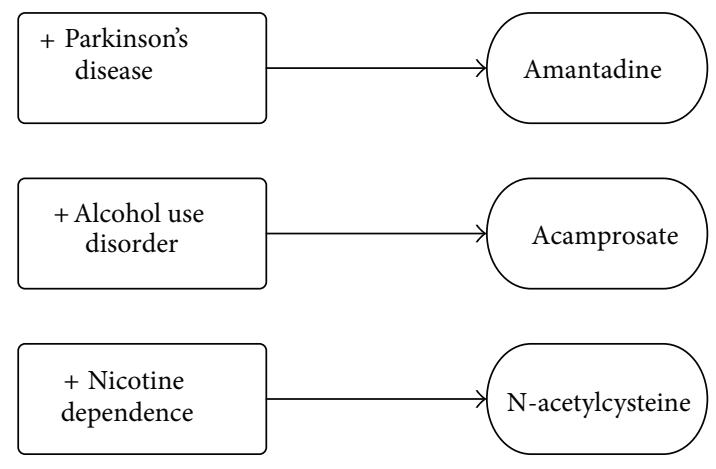

(b)

FIGURE 2: Clinical domains and comorbidity issues in the selection of glutamatergic treatment strategies to treat pathological gambling.

to regulate response to opioid antagonists across diagnostic groups [68]. Similarly, future studies should investigate the biological and psychological features of PG patients for whom glutamatergic treatment is appropriate. Based on current knowledge, we suggest clinical domains and comorbidity issues that may help guide the clinicians in the selection of appropriate glutamatergic treatment strategies (Figure 2). This model may provide the basis and rationale to guide the selection of pharmacotherapies in some groups of PG patients. Further investigations are certainly needed to confirm the treatment algorithm we propose.

Following cocaine administration, disrupted Glu homeostasis of nucleus accumbens core has been observed. A hallmark of disrupted homeostasis is a decrease in expression and function of the major Glu transporter, GLT-1 [69]. Future studies should investigate its role in PG and the potential utility of drugs that act to modulate the expression of Glu neurotransmitter transporters via gene activation (i.e., ceftriaxone) [70].

Besides Glu and DA, other factors, like brain-derived neurotrophic factor (BDNF), can be involved in the action of glutamatergic agents in PG [71]. Neurotrophic factors have been shown to be modulated by environmental events in various psychopathological conditions [72], and their role has been confirmed in the pathophysiology of PG [73]. Future studies should help understand the potential role of glutamatergic modulation on neurotrophins levels in PG patients.

Future investigations would benefit from placebo-controlled clinical trials to outline the true benefits of glutamatergic drugs for the treatment of PG. In addition, future research could profit from pharmacological challenges in combination with neuroimaging techniques to shed light on Glu role in the pathophysiology of PG. New neurobiological PG research should include matched controls, account for comorbidity issues, and differentiate between gambling preferences. Investigations in specific subgroups, therefore, are expected to give more insight into the pathophysiology of the disorder in these groups and perhaps lead to more tailored and efficient therapies. Future studies should also focus on the functional connections between dopaminergic and glutamatergic systems, in order to shed light upon the complex neurobiological mechanisms underlying the development of maladaptive gambling behavior.

\section{Abbreviations}

$\begin{array}{ll}\text { PG: } & \text { Pathological gambling } \\ \text { Glu: } & \text { Glutamate } \\ \text { DA: } & \text { Dopamine } \\ \text { NMDA: } & \text { N-methyl-d-aspartate } \\ \text { AMPA: } & \begin{array}{l}\alpha \text {-amino-3-hydroxy-5-methyl-4-isoazole- } \\ \text { propionic acid }\end{array} \\ \text { GABA: } & \text { Gamma-aminobutyric acid } \\ \text { CSF: } & \text { Cerebrospinal fluid } \\ \text { NAC: } & \text { N-acetylcysteine } \\ \text { RCT: } & \text { Randomized-controlled trial } \\ \text { PG-YBOCS: } & \text { Yale-Brown Obsessive Compulsive Scale } \\ & \text { modified for PG } \\ \text { G-SAS: } & \text { Gambling Severity Assessment Scale. }\end{array}$

\section{Conflict of Interests}

The authors declare that there is no conflict of interests regarding the publication of this paper.

\section{References}

[1] D. C. Hodgins, J. N. Stea, and J. E. Grant, "Gambling disorders," The Lancet, vol. 378, no. 9806, pp. 1874-1884, 2011.

[2] P. W. Kalivas, "The glutamate homeostasis hypothesis of addiction," Nature Reviews Neuroscience, vol. 10, no. 8, pp. 561-572, 2009.

[3] E. M. Krupitsky, A. A. Rudenko, A. M. Burakov et al., "Antiglutamatergic strategies for ethanol detoxification: comparison with placebo and diazepam," Alcoholism: Clinical and Experimental Research, vol. 31, no. 4, pp. 604-611, 2007.

[4] S. Rösner, S. Leucht, P. Lehert, and M. Soyka, "Acamprosate supports abstinence, naltrexone prevents excessive drinking: evidence from a meta-analysis with unreported outcomes," Journal of Psychopharmacology, vol. 22, no. 1, pp. 11-23, 2008.

[5] M. N. Potenza, "The neurobiology of pathological gambling and drug addiction: an overview and new findings," Philosophical Transactions of the Royal Society B: Biological Sciences, vol. 363, no. 1507, pp. 3181-3189, 2008.

[6] D. W. Black, D. P. McNeilly, W. J. Burke, M. C. Shaw, and J. Allen, "An open-label trial of acamprosate in the treatment of pathological gambling," Annals of Clinical Psychiatry, vol. 23, no. 4, pp. 250-256, 2011. 
[7] P. N. Dannon, O. Rosenberg, N. Schoenfeld, and M. Kotler, "Acamprosate and baclofen were not effective in the treatment of pathological gambling: preliminary blind rater comparison study," Frontiers in Psychiatry, vol. 2, article 33, 2011.

[8] M. Pettorruso, G. Martinotti, M. di Nicola et al., "Amantadine in the treatment of pathological gambling: a case report," Frontiers in Psychiatry, vol. 3, article 102, 2012.

[9] A. Thomas, L. Bonanni, F. Gambi, A. Di Iorio, and M. Onofrj, "Pathological gambling in parkinson disease is reduced by amantadine," Annals of Neurology, vol. 68, no. 3, pp. 400-404, 2010.

[10] M. Pettorruso, G. Conte, E. Righino et al., "2876-Glutamatergic strategies in the treatment of pathological gambling: a pilot study," European Psychiatry, vol. 28, supplement 1, 1 page, 2013.

[11] J. E. Grant, S. R. Chamberlain, B. L. Odlaug, M. N. Potenza, and S. W. Kim, "Memantine shows promise in reducing gambling severity and cognitive inflexibility in pathological gambling: a pilot study," Psychopharmacology, vol. 212, no. 4, pp. 603-612, 2010.

[12] Z. M. Pavlovic, "Psychopharmacological treatment of obsessive-compulsive disorder comorbid with body dysmorphic disorder and pathological gambling disorder," Journal of Neuropsychiatry and Clinical Neurosciences, vol. 23, no. 3, pp. E42-E43, 2011.

[13] M. Zack and C. X. Poulos, "Effects of the atypical stimulant modafinil on a brief gambling episode in pathological gamblers with high vs. low impulsivity," Journal of Psychopharmacology, vol. 23, no. 6, pp. 660-671, 2009.

[14] J. E. Grant, S. W. Kim, and B. L. Odlaug, "N-acetyl cysteine, a glutamate-modulating agent, in the treatment of pathological gambling: a pilot study," Biological Psychiatry, vol. 62, no. 6, pp. 652-657, 2007.

[15] J. E. Grant, B. L. Odlaug, S. R. Chamberlain et al., "A randomized, placebo-controlled trial of $\mathrm{N}$-acetylcysteine plus imaginal desensitization for nicotine-dependent pathological gamblers," The Journal of Clinical Psychiatry, vol. 75, no. 1, pp. 39-45, 2014.

[16] P. N. Dannon, K. Lowengrub, Y. Gonopolski, E. Musin, and M. Kotler, "Topiramate versus fluvoxamine in the treatment of pathological gambling: a randomized, blind-rater comparison study," Clinical Neuropharmacology, vol. 28, no. 1, pp. 6-10, 2005.

[17] H. A. Berlin, A. Braun, D. Simeon et al., "A double-blind, placebo-controlled trial of topiramate for pathological gambling," World Journal of Biological Psychiatry, vol. 14, no. 2, pp. 121-128, 2013.

[18] R. Nicolato, M. A. Romano-Silva, H. Correa, J. V. Salgado, and A. L. Teixeira, "Lithium and topiramate association in the treatment of comorbid pathological gambling and bipolar disorder," The Australian and New Zealand Journal of Psychiatry, vol. 41, no. 7, pp. 628-629, 2007.

[19] R. Dingledine, K. Borges, D. Bowie, and S. F. Traynelis, "The glutamate receptor ion channels," Pharmacological Reviews, vol. 51, no. 1, pp. 7-61, 1999.

[20] P. J. Conn and J.-P. Pin, "Pharmacology and functions of metabotropic glutamate receptors," Annual Review of Pharmacology and Toxicology, vol. 37, pp. 205-237, 1997.

[21] P. J. Kenny and A. Markou, "The ups and downs of addiction: role of metabotropic glutamate receptors," Trends in Pharmacological Sciences, vol. 25, no. 5, pp. 265-272, 2004.

[22] T. M. Tzschentke and W. J. Schmidt, "Glutamatergic mechanisms in addiction," Molecular Psychiatry, vol. 8, no. 4, pp. 373382, 2003.
[23] J. L. Cornish and P. W. Kalivas, "Cocaine sensitization and craving: differing roles for dopamine and glutamate in the nucleus accumbens," Journal of Addictive Diseases, vol. 20, no. 3, pp. 43$54,2001$.

[24] C. Heidbreder, "Novel pharmacotherapeutic targets for the management of drug addiction," European Journal of Pharmacology, vol. 526, no. 1-3, pp. 101-112, 2005.

[25] M. Melis, S. Spiga, and M. Diana, "The dopamine hypothesis of drug addiction: hypodopaminergic state," International Review of Neurobiology, vol. 63, pp. 101-154, 2005.

[26] K. C. Berridge and T. E. Robinson, "What is the role of dopamine in reward: hedonic impact, reward learning, or incentive salience?" Brain Research Reviews, vol. 28, no. 3, pp. 309-369, 1998.

[27] M. N. Potenza, "How central is dopamine to pathological gambling or gambling disorder?" Frontiers in Behavioral Neuroscience, vol. 7, article 206, 2013.

[28] M. Pettorruso, G. Martinotti, A. Fasano et al., "Anhedonia in Parkinson's disease patients with and without pathological gambling: a case-control study," Psychiatry Research, vol. 215, no. 2, pp. 448-452, 2014.

[29] R. J. van Holst, W. van den Brink, D. J. Veltman, and A. E. Goudriaan, "Why gamblers fail to win: a review of cognitive and neuroimaging findings in pathological gambling," Neuroscience and Biobehavioral Reviews, vol. 34, no. 1, pp. 87-107, 2010.

[30] K. McFarland, C. C. Lapish, and P. W. Kalivas, "Prefrontal glutamate release into the core of the nucleus accumbens mediates cocaine-induced reinstatement of drug-seeking behavior," Journal of Neuroscience, vol. 23, no. 8, pp. 3531-3537, 2003.

[31] R. T. LaLumiere and P. W. Kalivas, "Glutamate release in the nucleus accumbens core is necessary for heroin seeking," Journal of Neuroscience, vol. 28, no. 12, pp. 3170-3177, 2008.

[32] K. McFarland and P. W. Kalivas, "The circuitry mediating cocaine-induced reinstatement of drug-seeking behavior," Journal of Neuroscience, vol. 21, no. 21, pp. 8655-8663, 2001.

[33] C. Nordin, R. C. Gupta, and I. Sjödin, "Cerebrospinal fluid amino acids in pathological gamblers and healthy controls," Neuropsychobiology, vol. 56, no. 2-3, pp. 152-158, 2007.

[34] P. W. Kalivas and N. D. Volkow, "New medications for drug addiction hiding in glutamatergic neuroplasticity," Molecular Psychiatry, vol. 16, no. 10, pp. 974-986, 2011.

[35] M. F. Olive, R. M. Cleva, P. W. Kalivas, and R. J. Malcolm, "Glutamatergic medications for the treatment of drug and behavioral addictions," Pharmacology Biochemistry and Behavior, vol. 100, no. 4, pp. 801-810, 2012.

[36] R. Machado-Vieira, L. Ibrahim, I. D. Henter, and C. A. Zarate Jr., "Novel glutamatergic agents for major depressive disorder and bipolar disorder," Pharmacology Biochemistry and Behavior, vol. 100, no. 4, pp. 678-687, 2012.

[37] M. di Nicola et al., "Bipolar disorder and gambling disorder comorbidity: current evidence and implications for pharmacological treatment," Journal of Affective Disorders. In press.

[38] D. A. Baker, K. McFarland, R. W. Lake et al., "Neuroadaptations in cystine-glutamate exchange underlie cocaine relapse," Nature Neuroscience, vol. 6, no. 7, pp. 743-749, 2003.

[39] M. M. Moran, K. McFarland, R. I. Melendez, P. W. Kalivas, and J. K. Seamans, "Cystine/glutamate exchange regulates metabotropic glutamate receptor presynaptic inhibition of excitatory transmission and vulnerability to cocaine seeking," Journal of Neuroscience, vol. 25, no. 27, pp. 6389-6393, 2005. 
[40] D. A. Baker, K. McFarland, R. W. Lake, H. Shen, S. Toda, and P. W. Kalivas, "N-acetyl cysteine-induced blockade of cocaineinduced reinstatement," Annals of the New York Academy of Sciences, vol. 1003, pp. 349-351, 2003.

[41] Y. M. Kupchik, K. Moussawi, X.-C. Tang et al., "The effect of Nacetylcysteine in the nucleus accumbens on neurotransmission and relapse to cocaine," Biological Psychiatry, vol. 71, no. 11, pp. 978-986, 2012.

[42] P. W. Kalivas and C. O'Brien, "Drug addiction as a pathology of staged neuroplasticity," Neuropsychopharmacology, vol. 33, no. 1, pp. 166-180, 2008.

[43] G. Sani, G. Serra, G. D. Kotzalidis et al., "The role of memantine in the treatment of psychiatric disorders other than the dementias: a review of current preclinical and clinical evidence," CNS Drugs, vol. 26, no. 8, pp. 663-690, 2012.

[44] H. Van Wageningen, H. A. Jørgensen, K. Specht, and K. Hugdahl, "A 1H-MR spectroscopy study of changes in glutamate and glutamine (Glx) concentrations in frontal spectra after administration of memantine," Cerebral Cortex, vol. 20, no. 4, pp. 798803, 2010.

[45] R. F. Leeman and M. N. Potenza, "Similarities and differences between pathological gambling and substance use disorders: a focus on impulsivity and compulsivity," Psychopharmacology, vol. 219, no. 2, pp. 469-490, 2012.

[46] A. Fasano, L. Ricciardi, M. Pettorruso, and A. R. Bentivoglio, "Management of punding in Parkinson's disease: an open-label prospective study," Journal of Neurology, vol. 258, no. 4, pp. 656660, 2011.

[47] K. Seppi, D. Weintraub, M. Coelho et al., "The movement disorder society evidence-based medicine review update: treatments for the non-motor symptoms of Parkinson's disease," Movement Disorders, vol. 26, no. 3, pp. S42-S80, 2011.

[48] D. Weintraub, M. Sohr, M. N. Potenza et al., "Amantadine use associated with impulse control disorders in Parkinson disease in cross-sectional study," Annals of Neurology, vol. 68, no. 6, pp. 963-968, 2010.

[49] J. T. Gass and M. F. Olive, "Glutamatergic substrates of drug addiction and alcoholism," Biochemical Pharmacology, vol. 75, no. 1, pp. 218-265, 2008.

[50] P. De Witte, J. Littleton, P. Parot, and G. Koob, "Neuroprotective and abstinence-promoting effects of acamprosate: elucidating the mechanism of action," CNS Drugs, vol. 19, no. 6, pp. 517-537, 2005.

[51] G. Rammes, B. Mahal, J. Putzke et al., "The anti-craving compound acamprosate acts as a weak NMDA-receptor antagonist, but modulates NMDA-receptor subunit expression similar to memantine and MK-801," Neuropharmacology, vol. 40, no. 6, pp. 749-760, 2001.

[52] R. Spanagel, V. Vengeliene, B. Jandeleit et al., "Acamprosate produces its anti-relapse effects via calcium," Neuropsychopharmacology, vol. 39, no. 4, pp. 783-791, 2014.

[53] F. Kiefer and K. Mann, "Acamprosate: how, where, and for whom does it work? Mechanism of action, treatment targets, and individualized therapy," Current Pharmaceutical Design, vol. 16, no. 19, pp. 2098-2102, 2010.

[54] L. A. Boothby and P. L. Doering, "Acamprosate for the treatment of alcohol dependence," Clinical Therapeutics, vol. 27, no. 6, pp. 695-714, 2005.

[55] Y. P. Raj, “Gambling on acamprosate: a case report," Journal of Clinical Psychiatry, vol. 71, no. 9, pp. 1245-1246, 2010.
[56] J. R. Chalifoux and A. G. Carter, "GABAB receptor modulation of synaptic function," Current Opinion in Neurobiology, vol. 21, no. 2, pp. 339-344, 2011.

[57] G. Addolorato, L. Leggio, S. Cardone, A. Ferrulli, and G. Gasbarrini, "Role of the GABAB receptor system in alcoholism and stress: focus on clinical studies and treatment perspectives," Alcohol, vol. 43, no. 7, pp. 559-563, 2009.

[58] F. A. Furieri and E. M. Nakamura-Palacios, "Gabapentin reduces alcohol consumption and craving: a randomized, doubleblind, placebo-controlled trial," Journal of Clinical Psychiatry, vol. 68, no. 11, pp. 1691-1700, 2007.

[59] G. Martinotti, "Pregabalin in clinical psychiatry and addiction: pros and cons," Expert Opinion on Investigational Drugs, vol. 21, no. 9, pp. 1243-1245, 2012.

[60] I. Cuomo, G. D. Kotzalidis, F. Caccia, E. Danese, G. Manfredi, and P. Girardi, "Citalopram-associated gambling: a case report," Journal of Gambling Studies, vol. 30, no. 2, pp. 467-473, 2014.

[61] J. S. Ballon and D. Feifel, "A systematic review of modafinil: potential clinical uses and mechanisms of action," Journal of Clinical Psychiatry, vol. 67, no. 4, pp. 554-566, 2006.

[62] J. Martínez-Raga, C. Knecht, and S. Cepeda, "Modafinil: a useful medication for cocaine addiction? Review of the evidence from neuropharmacological, experimental and clinical studies," Current drug abuse reviews, vol. 1, no. 2, pp. 213-221, 2008.

[63] N. D. Volkow, J. S. Fowler, J. Logan et al., "Effects of modafinil on dopamine and dopamine transporters in the male human brain clinical implications," Journal of the American Medical Association, vol. 301, no. 11, pp. 1148-1154, 2009.

[64] L. Ferraro, T. Antonelli, W. T. O'Connor, S. Tanganelli, F. Rambert, and K. Fuxe, "The antinarcoleptic drug modafinil increases glutamate release in thalamic areas and hippocampus," NeuroReport, vol. 8, no. 13, pp. 2883-2887, 1997.

[65] K. Smart, R. C. Desmond, C. X. Poulos, and M. Zack, "Modafinil increases reward salience in a slot machine game in low and high impulsivity pathological gamblers," Neuropharmacology, vol. 73, pp. 66-74, 2013.

[66] N. Tarrant, A. E. Cavanna, and H. Rickards, "Pathological gambling associated with modafinil," Journal of Neuropsychiatry and Clinical Neurosciences, vol. 22, no. 1, pp. E27-E28, 2010.

[67] R. Ladouceur, C. Sylvain, C. Boutin, S. Lachance, C. Doucet, and J. Leblond, "Group therapy for pathological gamblers: a cognitive approach," Behaviour Research and Therapy, vol. 41, no. 5, pp. 587-596, 2003.

[68] J. E. Grant, S. W. Kim, E. Hollander, and M. N. Potenza, "Predicting response to opiate antagonists and placebo in the treatment of pathological gambling," Psychopharmacology, vol. 200, no. 4, pp. 521-527, 2008.

[69] K. Tanaka, K. Watase, T. Manabe et al., "Epilepsy and exacerbation of brain injury in mice lacking the glutamate transporter GLT-1," Science, vol. 276, no. 5319, pp. 1699-1702, 1997.

[70] Y. Sari, M. Sakai, J. M. Weedman, G. V. Rebec, and R. L. Bell, "Ceftriaxone, a beta-lactam antibiotic, reduces ethanol consumption in alcohol-preferring rats," Alcohol and Alcoholism, vol. 46, no. 3, Article ID agr023, pp. 239-246, 2011.

[71] J. Jeanblanc, F. Coune, B. Botia, and M. Naassila, "Brain-derived neurotrophic factor mediates the suppression of alcohol selfadministration by memantine," Addiction Biology, 2013.

[72] F. Angelucci, V. Ricci, F. Gelfo et al., "BDNF serum levels in subjects developing or not post-traumatic stress disorder after trauma exposure," Brain and Cognition, vol. 84, no. 1, pp. 118122, 2014. 
[73] F. Angelucci, G. Martinotti, F. Gelfo et al., "Enhanced BDNF serum levels in patients with severe pathological gambling," Addiction Biology, vol. 18, no. 4, pp. 749-751, 2013. 

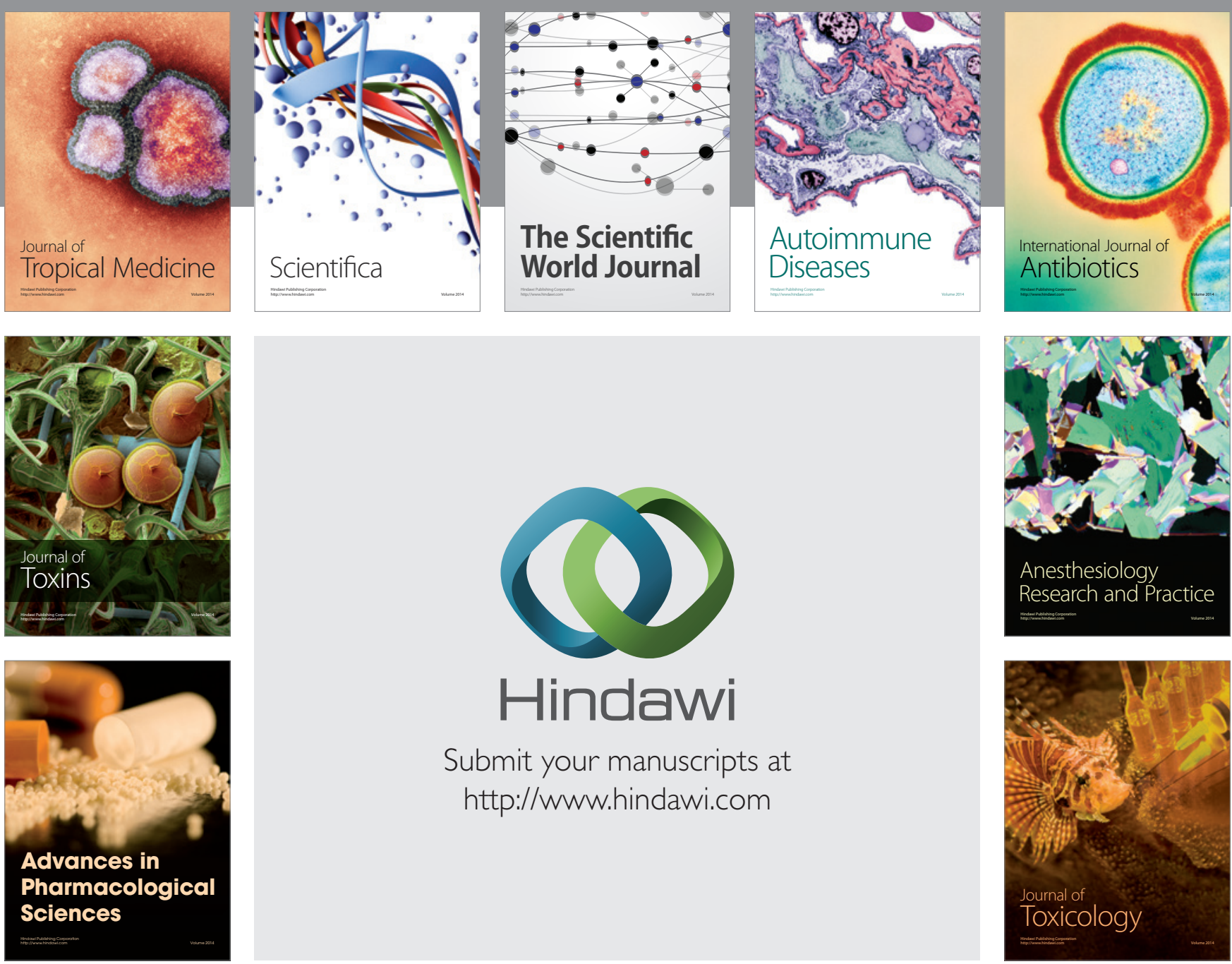

\section{Hindawi}

Submit your manuscripts at

http://www.hindawi.com
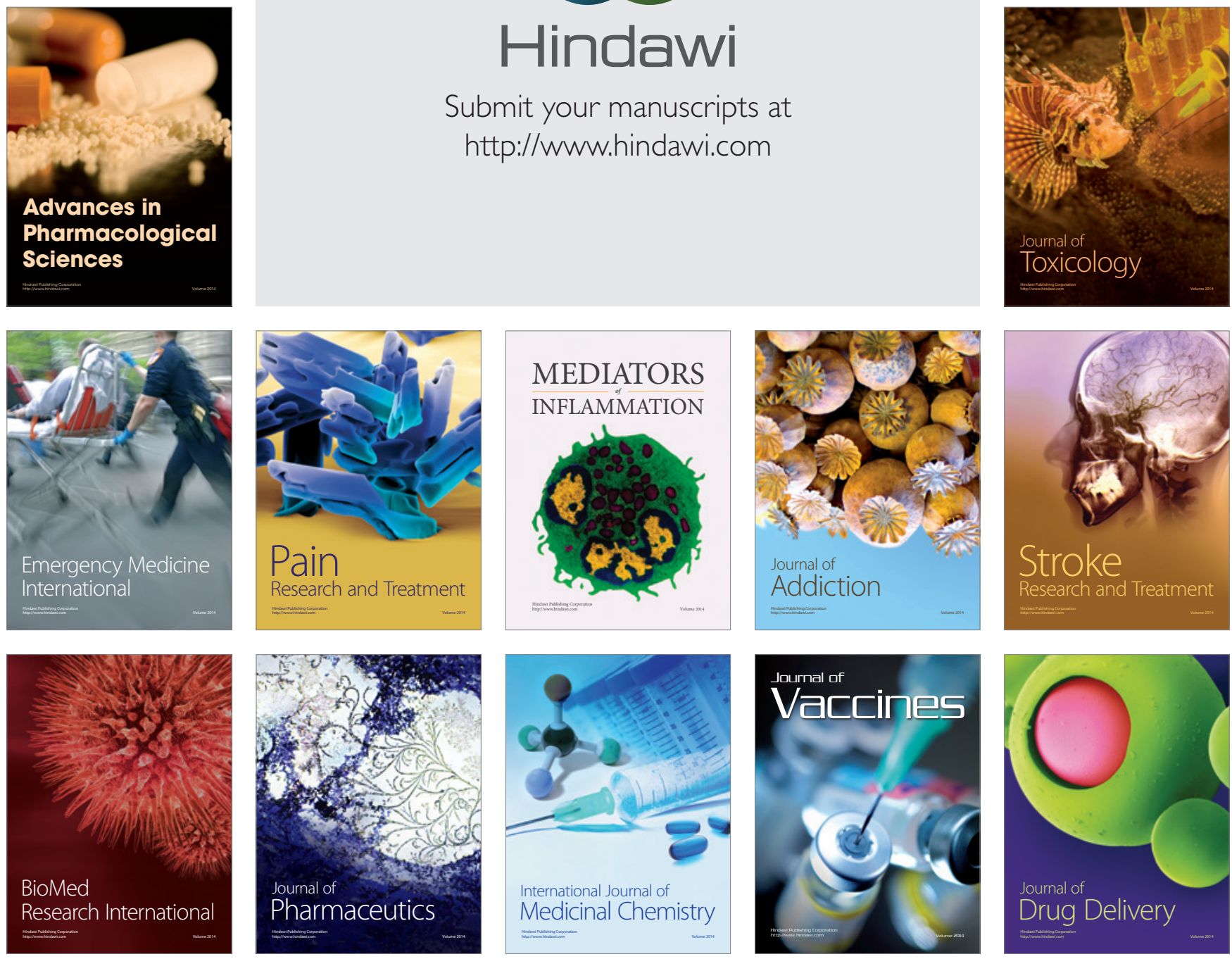